\title{
QUANTITATIVE FLOW CHARACTERISTICS FOR SIDE-BY-SIDE SQUARE CYLINDERS VIA PIV
}

\begin{abstract}
Muammer OZGOREN ${ }^{1}$ and Sercan DOGAN ${ }^{2}$
Abstract: In this study, instantaneous and time-averaged flow structures downstream of the sharp-edged single and two and three side-by-side square cylinders (SCs) immersed in a uniform open channel water flow were studied by a technique of particle image velocimetry (PIV). Experimental results of wake flow structures were presented for gap ratios $(G / D)$ in the range of $1.0 \leq G / D \leq 3.0$ for Reynolds number values of 1050, 2450 and 3400. Flow structures depending on the square cylinder (SC) configurations and Reynolds number were discussed. It has been found that the development of the vortex shedding as well as the flow structure were substantially altered for side-by-side SCs comparing to the single SC. Asymmetrical and biased wake structures were observed because of the jetlike flow between the SCs for two SCs cases for the gap ratio less than 2.0. Depending on the gap spacing between the SCs, the interaction results of timeaveraged vorticity, velocity vector field, Reynolds stress correlations $\left\langle\mathrm{u}^{\prime} \mathrm{v}^{\prime} / \mathrm{U}_{\infty}^{2}\right\rangle$ and streamline patterns in the wake region form a distinguished flow structure. Strouhal numbers for the single square cylinder for $1050 \leq R e \leq 3400$ are found in the range of 0.12-0.13. The present results have supported the previous works by providing detailed quantitative experimental information with PIV in the wake region of the SC and might be helpful for validation of numerical studies and designers.
\end{abstract}

Keywords: Coupled vortex, instability, PIV, side-by-side square cylinders, Strouhal numbers, vortex shedding, wake flow, turbulence

\section{INTRODUCTION}

The subject of single, two or multiple bluff-body flows owing to interactions of flow and solid structure has been attracted a great deal of attention, recently. The knowledge of the flow field around bluff bodies is of major importance in many engineering applications including cooling of electronic equipments, heat exchangers, groups of neighboring tall buildings, chimneys, bridges and trash racks. One of the main features of these bluff body flow configurations is a periodic force loading in streamwise and vertical directions due to the pressure variations on the cylinder surface caused by periodic vortex shedding. When more than one square cylinder (SC) are placed in a uniform flow, the flow characteristics such as surface pressure, drag coefficient and vortex-shedding frequency are completely different from the case of single SC depending on the arrangement of the gap ratio G/D of the SCs and Reynolds number. Here, $G$ is the distance between the center of the SCs and D is the width of the SC. In addition, the flow structure around two or three SCs displays a very complicated flow patterns due to the

\footnotetext{
${ }^{1}$ Selcuk University, Engineering and Architecture Faculty, Department of Mechanical Engineering, , 42250, Konya, Turkey, email: mozgoren@selcuk.edu.tr

${ }^{2}$ Research Assistant, Engineering and Architecture Faculty,Mechanical Engineering

Department, email: sercandogan@selcuk.edu.tr
} 
strong interference of vortices caused by the SCs when the gap ratio G/D is relatively short. For this reason, aerodynamic forces acting on the two or three structures could be very different in comparison with a single structure [1].

A large number of studies have been performed to investigate the flow characteristics of single SC and circular cylinder (CC) [1-6]. Okajima (1982) carried out an experimental study of flow past a SC as well as rectangular cylinder for $70 \leq R e \leq 20,000$ to determine the vortex shedding frequencies. The results showed that there was an abrupt change in Strouhal number when the aspect ratio of the cylinder was reduced to the range of 2-3 [2]. Obasaju (1983) examined Strouhal numbers for the SC with increasing an angle of incidence from $0^{\circ}$ to $45^{\circ}$ using a hot-wire probe in a closed circuit wind tunnel for $\operatorname{Re}=10,000$ [3]. Laser Doppler Velocimetry (LDV) measurements for the flow past a SC in a water tunnel for $\operatorname{Re}=21,400$ were conducted [4]. They showed the relationship between the flow patterns and turbulence distribution. Measurements of two-components of velocity in the wake of the SC for Reynolds numbers of 1340, 4990 and 9980 having various inclinations such as $0^{\circ}$, $22.5^{\circ}, 30^{\circ}, 45^{\circ}$, and $60^{\circ}$ using smoke visualization and a hot-wire anemometer were reported [5]. They stated that although the overall flow structure past the SC resembled that of the CC, there were major differences as far as the separation mechanism and the related integral parameters such as Strouhal number, lift and drag-coefficient concerned. Ozgoren (2006) visualized flow characteristics quantitatively in the downstream region of the single circular, sharp-edged square and $45^{\circ}$ orientated SC in a uniform flow using PIV [6]. Many interesting and unexpected fluid phenomena may occur when two or more bluff bodies are placed one beside the other as reported by Zdravkovich [7] and Williamson [1]. The grouping effect of bodies is a very interesting subject of fundamental research in fluid mechanics. In the open literature, there is not much research work dealing with side-by-side SCs; however, most of researches carried out to understand the flow structure around sideby-side circular cylinders (CCs). The case of a steady flow passing two or more CCs in a side-by-side arrangement have been investigated by many researcher [1,7-14]. Williamson (1985) investigated the flow structure behind a pair of the CCs placed side-by-side at $\mathrm{Re}$ $=50-150,200$ and $1.5<G / D<5.0$ using flow-visualization methods [1]. He observed that vortex shedding synchronization between two CCs wakes occurred either in phase or in antiphase modes when the gap spacing exceeded for one cylinder diameter. He displayed that below a critical gap between the CCs the flow became asymmetric, similar to the results found by Bearman \& Wadcock [8]. Kim and Durbin (1988) examined the unsteady wake arising for the gap spacing less than one diameter, for $R e=2000-7000$ and $G / D=1.75$ experimentally. The deflected gap flow can be bistable, that is, the deflection can change its direction in a random way and stay in the same direction for a while [8]. They reported that the bistability was nominally independent of the Reynolds number for CCs [8]. Guillaume and LaRue (1999) presented the variation of the base pressure coefficients and the characteristics of the velocity power spectra for arrays of two, three, and four-CCs aligned normal to the flow [10]. They used hot-wire sensors and smoke flow visualization to examine flopping behavior of the wake for $R e=2500$ and $1.0<G / D<2.2$. Sumner et al. (1999) investigated the flow patterns downstream of two and three side-by-side CCs for 1.0 $\leq \mathrm{G} / \mathrm{D} \leq 6.0$ and $\mathrm{Re}=500-3000$ using flow visualization and the PIV technique [11]. Zhou's research group extensively performed a series of experimental studies on the flow for CCs [12-13]. They investigated the turbulent wake characteristics at $G / D=1.5$ and $G / D=3.0$ for $\mathrm{Re}=5800$ around two and three side-by-side CCs. They stated that the mutual interactions between the narrow and wide wakes probably led to the early vanishing of the narrow wake for $G / D=1.5$ in the asymmetric flow regime.

Flows passing multiple SCs or flat plates, both of which have fixed separation points at the leading edge are extensively reported [15-19]. Bearman and Wadcock (1973) qualitatively measured different base pressures downstream of the two side-by-side flat plates and they 
expressed that the gap flow deflection was not caused by the boundary layer separation; instead, it was due to the near-wake phenomenon [8]. Sayers (1991) determined pressure distribution around three equal spaced SCs for side-by-side and tandem arrangements in the range of gap spacings $1.1<\mathrm{G} / \mathrm{D}<3.0$ for $\mathrm{Re}=30,000$ [15]. Depending on gap spacing, Sayers also noted large changes in the drag and lift coefficients for the transverse configuration downstream individual SC. Miau et al. (1994) observed irregular biasing gap in the downstream of two side-by-side flat plates [16]. Higuchi et al. (1994) demonstrated the nature of the non-periodic and multi scale vortex shedding behind a pair of flat plates, particularly on the wide wake side, from the results of wavelet and conditional spectral analysis. Their assessments revealed that when the distances between two SCs in side-byside arrangement were long, the computed time-averaged drag coefficients were larger than experimental data [17]. Kolar et al. (1997) studied ensemble-averaged characteristics of the turbulent near-wake flow around two side-by-side identical SCs for $G / D=3.0$ and $\operatorname{Re}=23,100$ using a two-component LDV system [18]. They stated that individual vortex streets were coupled yielding a flow predominantly symmetric about midway line between two SCs. Furthermore, they observed qualitatively biased flow regime for $1.5<\mathrm{G} / \mathrm{D}<2.0$ and coupled vortex street regime with in phase or in anti-phase mode for the range of $2.0<G / D<6.0$. Alam et al. (2003) investigated the characteristics of wake frequency, the switching phenomenon, and fluid forces acting on two CCs and SCs in a side-by-side arrangements with flow visualization and a point wise measurement technique for two $\mathrm{SCs}$ at $\mathrm{Re}=47,000$ in the range of $1.0<\mathrm{G} / \mathrm{D} \leq 5.0$ [21]. They reported that three modes of frequency which were high, intermediate, and low in the signals appeared in the range of $G / D=1.4$ to $G / D=2.9$. A dominant shedding frequency only took place in the wavelet analysis of power spectra for increasing the gap ratio further. Alam et al. (2010) compared flow characteristics around two square and circular cylinders by using different techniques, including hot wires, load cell, particle imaging velocimetry and laser-induced fluorescence flow visualization for $\operatorname{Re}=47000$. They observed that four distinct flow regimes and their corresponding gap spacing ranges were identified for the first time on the basis of the flow structure and the Strouhal number [22].

Quantitative visualizations of wake flow structures of side-by-side two and three SCs have not been examined in detail. The present investigation aims at interpreting the physical characteristics of the near-wake flow structures downstream of a single, two and three sideby-side SCs to provide informative data for both scientists and designers. Quantitative visualization of instantaneous and time-averaged wake structures as a function of Reynolds number in the range $1050 \leq R e \leq 3,400$ and gap ratios of $1.0 \leq G / D \leq 3.00$ is discussed.

\section{EXPERIMENTAL SETUP}

An overview of experimental system of three side-by-side SCs case is shown in Figure 1. Experiments were carried out in a closed-loop free surface water channel having a width of $\mathrm{W}=1,000 \mathrm{~mm}$, a length of $8,000 \mathrm{~mm}$ and height of free-surface $\mathrm{H}=750 \mathrm{~mm}$. It was preceded by a $2: 1$ contraction ratio and flow conditioning section, which provided a freestream turbulence intensity of less than $0.5 \%$ in the range of present Reynolds numbers $1050 \leq \operatorname{Re} \leq 3,400, \operatorname{Re}=\left(U_{\infty} D\right) / v$, based on the SC width. Here, $v$ is kinematic viscosity and $\mathrm{U}_{\infty}$ is free-stream velocity ranging from $55.5 \mathrm{~mm} / \mathrm{s}$ to $170 \mathrm{~mm} / \mathrm{s}$. Sharp-edged SC with 20 $\mathrm{mm} \times 20 \mathrm{~mm}$ was made of plexiglas. The PIV technique that can give information on the spatial distribution of flow quantities in unsteady flow fields was used. The laser sheet was located at $330 \mathrm{~mm}$ above the bottom surface of the channel while the water height, $\mathrm{h}_{\mathrm{w}}$ was $600 \mathrm{~mm}$ in all cases. Aspect ratios were 30 and 50 with respect to the submerged section and whole length of the SC, respectively. The solid blockage ratio of each SC was $2 \%$, having a maximum blockage of $6 \%$ for three SCs case in the case of water channel. Nd:Yag 
laser was used to generate a laser sheet perpendicular to the axis of the SC, and a CCD camera having a resolution of $1,024 \times 1,024$ pixels was used to record the images. The output of the laser was transmitted through a cylindrical lens, which generated the planar laser to illuminate metal-coated small particles in the water flow. The seeding particles with a diameter of $15 \mu \mathrm{m}$ in the flow were silver metallic coated, hollow plastic spheres. The specific gravity of the seeding particles was 1.10 . The densities of the particles and water were close enough so that the distribution of particles in suspension remained uniform for several hours.


\section{SIDE VIEW}

\section{CCD Camera}

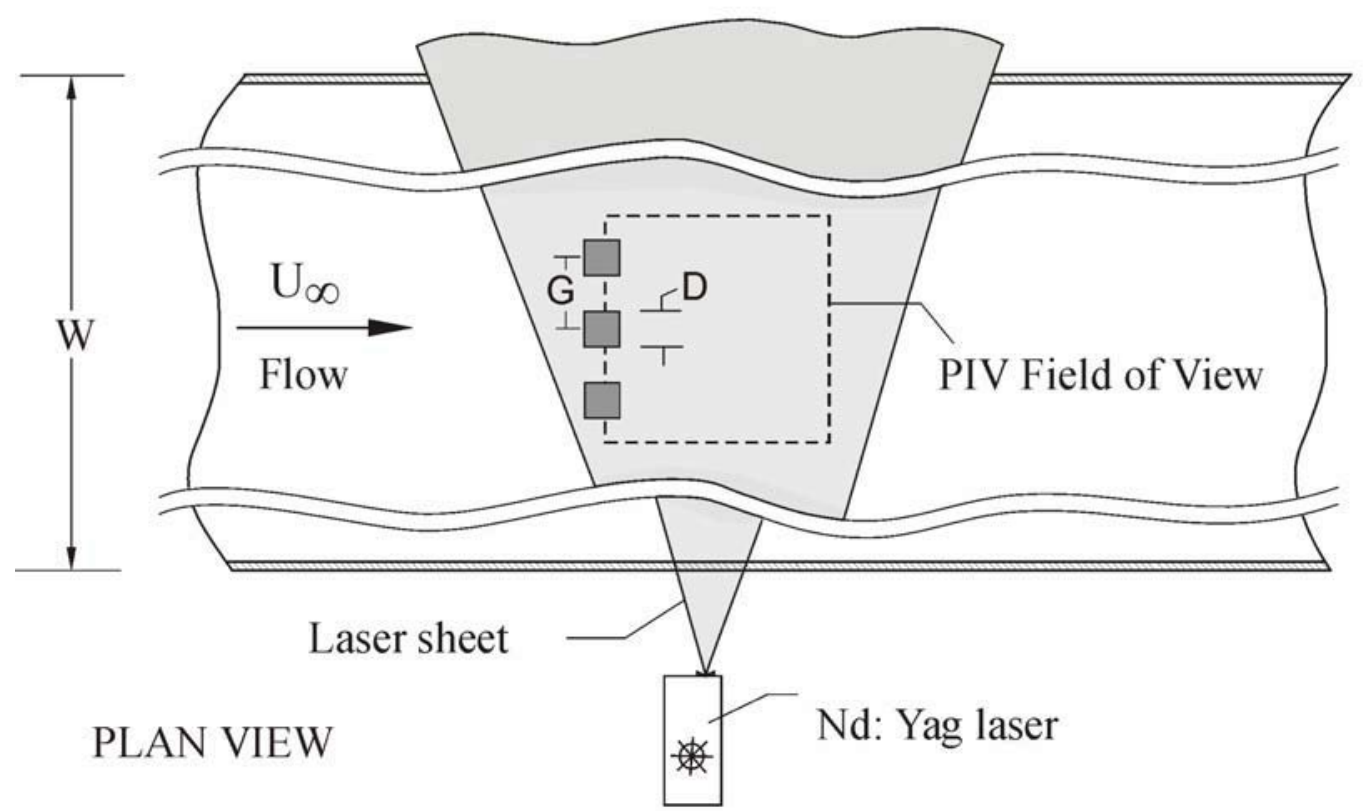

Figure 1: Schematic of the experimental system and definition of the parameters for three side-by-side SCs. 
The high-image-density criterion was satisfied by ensuring that a minimum of 20-30 particles was available within the interrogation area. The illuminating laser sheet thickness in the flow field was approximately $1.5 \mathrm{~mm}$. As shown in Figure 1, the camera was mounted in a fixed position beneath the water tank. Dantec Flow Grabber digital PIV software employing the cross-correlation algorithm was used to compute the raw displacement vector field from the particle image data, using an interrogation window of $32 \times 32$ pixels, approximately $2.75 \times 2.75 \mathrm{~mm}^{2}(0.138 \mathrm{D} \times 0.138 \mathrm{D})$. For whole experiments, the image magnification was $1: 5.91$ and the overall field of view was $171 \times 171 \mathrm{~mm}^{2}(8.5 \mathrm{D} \times 8.5 \mathrm{D})$ leading to 3,844 velocity vectors. During the interrogation process, an overlap of $50 \%$ was employed in order to satisfy the Nyquist criterion. Patterns of instantaneous particle images (total of 200 images for a continuous series) were taken at the rate of $15 \mathrm{~Hz}$, thereby spanning $13.4 \mathrm{sec}$. Averaged patterns of the flow structure were calculated from all of the instantaneous images. The time delay between pulses was ranged from $1 \mathrm{~ms}$ to $12 \mathrm{~ms}$. Inappropriate displacement vectors caused by shadows, reflections, or laser sheet distortions in the flow field replaced by using bilinear interpolation between surrounding vectors in the postprocessing step. This algorithm included magnification factor and image captured rate to calculate velocities from the valid vectors. The field was then smoothed by a Gaussian weighted averaging technique. To minimize distortion of the velocity field, a smoothing parameter of 1.3 was chosen. The vorticity patterns of the wake flow were determined from the velocity field using a finite difference scheme. The factors contributing to uncertainty of velocity measurement using the PIV technique were critically assessed and concluded that uncertainty estimation in the velocity measurement was less than $2 \%$ by Westerweel [23].

\section{RESULTS AND DISCUSSION}

\subsection{Instantaneous and Time-averaged flow structures for a single square cylinder}

Representative instantaneous velocity fields $V$ and vorticity contours $\omega$ of the wake structure for the sphere geometries are illustrated in Figure. 3. Viewing the vortex street in the measuring plane, concentrated vorticity layers $\omega$ are shed at the corner od the square cylinder, giving the appearance of negative vortices in clockwise direction (dashed lines) and positive vortices in anticlockwise direction (solid lines). The vortices $\omega$ produced from the flow separation have a tendency to move inwards because of the lower pressures prevailing within the wake. This situation is counter-balanced by the growing wake size, which shifted the vortex centerline outwards. Regarding the onset and development of small-scale vortical structures in the separating shear layer, regions of low-level vorticity concentrations are detectable in the pattern of instantaneous vorticity $\omega$ for all patterns in the first row of Figure 2 and in the first column of Figure 3. Large-scale Von Kármán vortices form in the near-wake region due to the abrupt coalescence of small-scale shear-layer vortices that developed along the separating shear layers similar to the results of Ozgoren (2006). Instantaneous vorticity patterns $\omega$ show irregular distributions, and the shear layers tend to break up easily into small concentrations of vorticity $\omega$ related to Kelvin-Helmholtz $(\mathrm{KH})$ instabilities. Concentrations of the small-scale vortices for the two side-by-side spheres are stronger than those for the single sphere case. The wake region accommodates velocity vectors $\mathrm{V}$ with very small magnitude in the downstream region of the SCs, which are the source of small-scale secondary vortices, as seen in the instantaneous flow patterns row in Figures 2 and 3 . Shedding vortices in the streamwise flow direction causes a successive vorticity peaks occurring close to each other in the near wake region for all cases. The flow is three-dimensional and shedding vortices convey fresh fluid into the wake flow region, magnifying the flow entrainment between core and wake flow regions. 
Comparison of flow structure for a square cylinder with instantaneous and time-averaged flow patterns are presented in Figure 2. Positive and negative flow patterns are displayed by solid and dashed lines, respectively. Instantaneous flow patterns in the first row of Figure shows the well known Karman Vortex streets. The natural frequency of shedding vortices occurred on both sides of bluff body is varied as a function of geometry and Reynolds number. The vortex shedding frequency from the SC is normalized by a Strouhal number, St $=f_{s} D / U_{\infty}$, where $f_{s}$ is the vortex shedding frequency. The Strouhal number is found to be 0.131 for $\mathrm{Re}=3400$. Present Strouhal numbers for $1050<\mathrm{Re}<3400$ have a good agreement with the results of Okajima $[2,5-6]$.
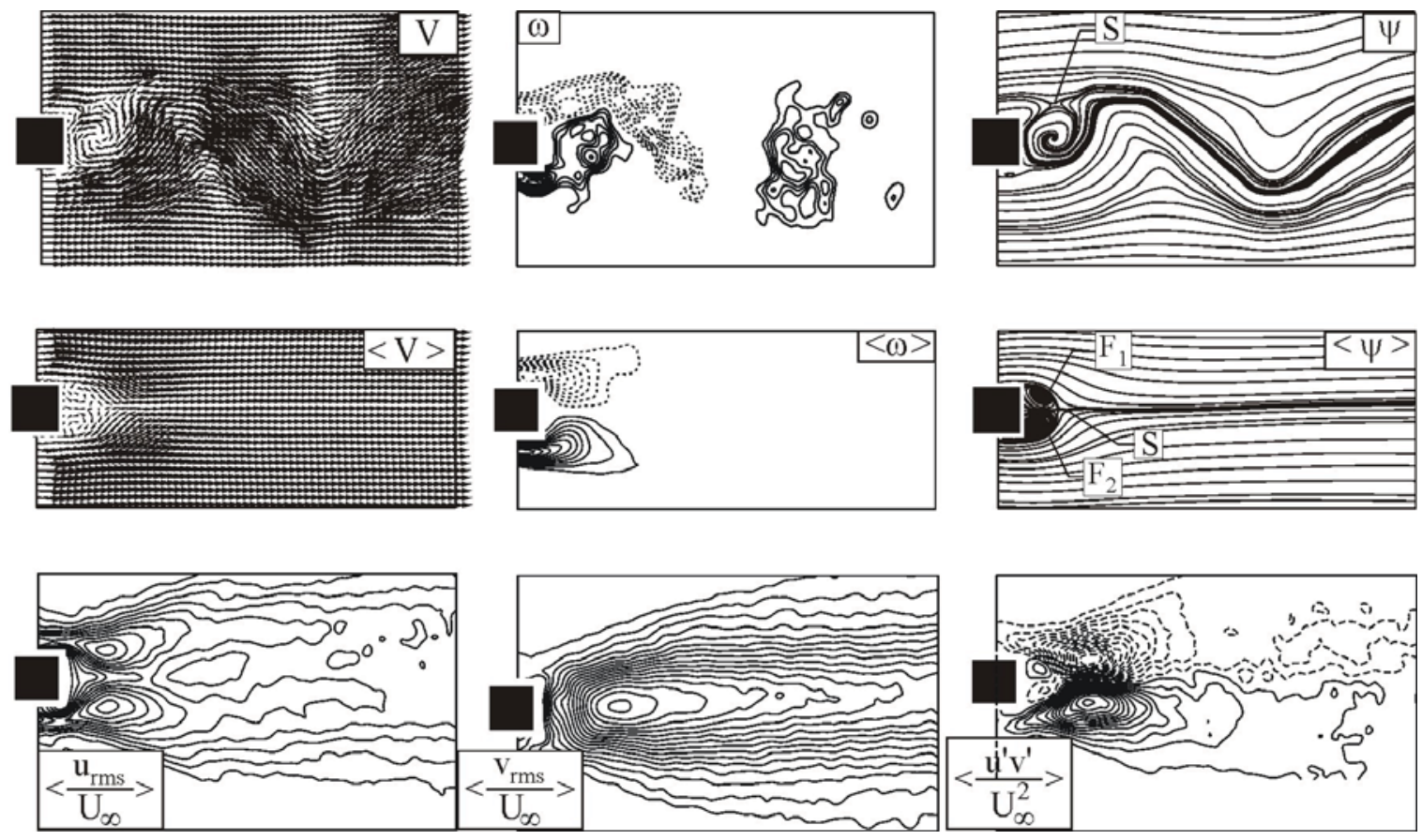

Figure 2: Comparison of patterns of instantaneous velocity field $V$, vorticity $\omega$, and streamline patterns $\psi$ together with time-averaged velocity fields $\langle\mathrm{V}\rangle$, vorticity $\langle\omega\rangle$, streamline patterns $\langle\psi\rangle$, dimensionless root-mean-square of the streamwise velocity fluctuations $\left\langle\mathrm{u}_{\mathrm{rms}} / \mathrm{U}_{\infty}\right\rangle$, cross-stream velocity $\left\langle\mathrm{v}_{\mathrm{rms}} / \mathrm{U}_{\infty}\right\rangle$ and Reynolds stress correlations $\left\langle\mathrm{u}^{\prime} \mathrm{v}^{\prime} / \mathrm{U}_{\infty}^{2}>\right.$ at $\mathrm{Re}=3400$ for a square cylinder. Minimum and incremental contour levels of parameters are $\omega_{\min }=\Delta \omega= \pm 5.0 \mathrm{~s}^{-1}$ for instantaneous vorticity, $\left\langle\omega_{\min }\right\rangle=\langle\Delta \omega\rangle= \pm 3.0 \mathrm{~s}^{-1}$ for time-averaged vorticity $\langle\omega\rangle$, $\left.<\mathrm{u}_{\mathrm{rms}} / \mathrm{U}_{\infty}\right\rangle_{\min }=\left\langle\mathrm{v}_{\mathrm{rms}} / \mathrm{U}_{\infty}\right\rangle_{\min }=0.01$ and $\left\langle\Delta \mathrm{u}_{\mathrm{rms}} / \mathrm{U}_{\infty}\right\rangle=\left\langle\Delta \mathrm{v}_{\mathrm{rms}} / \mathrm{U}_{\infty}\right\rangle= \pm 0.05$ for streamwise $<\mathrm{u}_{\mathrm{rms}} / \mathrm{U}_{\infty}>$ and cross stream $\left\langle\mathrm{v}_{\mathrm{rms}} / \mathrm{U}_{\infty}\right\rangle$ velocity fluctuations and $\left.<\mathrm{u}^{\prime} \mathrm{v}^{\prime} / \mathrm{U}_{\infty}^{2}\right\rangle_{\min }=\left\langle\Delta \mathrm{u}^{\prime} \mathrm{v}^{\prime} / \mathrm{U}_{\infty}^{2}\right\rangle= \pm 0.01$ for Reynolds stress correlations $\left.<\mathrm{u}^{\prime} \mathrm{v}^{\prime} / \mathrm{U}_{\infty}^{2}\right\rangle$.

In addition, observations of cinema of instantaneous data shows that shedding vortices with opposite sign interact more directly and vigorously, resulting in substantial cancellation of vorticity in the cases of $\operatorname{Re}=3,400$ as seen in the second row of Figure 2 by time-averaged velocity field $\langle\mathrm{V}\rangle$ and vorticity $\langle\mathrm{W}\rangle$. As seen in the third row of Figure 1 , the maximum values of the streamwise velocity fluctuations $\left\langle\mathrm{u}_{\mathrm{rms}} / \mathrm{U}_{\infty}\right\rangle$ with double peaks appear are 
detectable along shear layers for the case of single sphere. However, the maximum value of cross-stream velocity fluctuations $\left\langle\mathrm{v}_{\mathrm{rms}} / \mathrm{U}_{\infty}\right\rangle$ in the wake region corresponding to the location of saddle point S. Reynolds stress correlations $\left\langle\mathrm{u}^{\prime} \mathrm{v}^{\prime} / \mathrm{U}_{\infty}^{2}\right\rangle$ in the right column of Figure 2 depict a set of extrema on either side of the wake centerline for as a result of the momentum transfer to the wake. A well-defined Reynolds stress patterns due to fluctuations in the shear layers, weaker Reynolds stress concentrations very close to the base of the SC occur because of the flow entrainment between core and wake flow regions. Entrainment takes place along the shear layers region giving rise to a circulatory motion within the separated flow leading to a mass and momentum transfer between the main and wake-flow regions. Reynolds stress contours for the SC have peak value of 0.132 . For further details, researcher should refer to Ozgoren [6] who has investigated detailed studies of the flow structures in the downstream of single circular, square and oriented square cylinders.

The time-averaged patterns of streamline patterns $\langle\psi\rangle$ is interpreted in terms of foci, $F$, and saddle points, $\mathrm{S}$ as displayed in the second row of Figure 2. Comparison of the timeaveraged patterns shows that flow structures of the wake are almost equally symmetric with respect to the centerline of the square cylinders. Saddle point $S$ shows the merging point of the shear layers emanating around the periphery of the sphere. Locations of foci designated with $F_{1}$ and $F_{2}$ that roll in the clockwise and in counter clockwise with respect to the square cylinder central axis exhibit well-defined critical points.

\subsection{Instantaneous and time-averaged flow structures for two side-by-side square}

\section{cylinders}

Comparison of instantaneous vorticity $\omega$, time-averaged vorticity contours $\langle\omega\rangle$, timeaveraged velocity field $\langle\mathrm{V}\rangle$, streamline patterns $\langle\mathrm{y}\rangle$ and Reynolds stress correlations $\left\langle\mathrm{u}^{\prime} \mathrm{v}^{\prime} / \mathrm{U}_{\infty}^{2}\right\rangle$ as a function of gap spacing ratio $\mathrm{G} / \mathrm{D}=1.0$ to 3.0 is presented for two side-by-side $\mathrm{SCs}$ at $\mathrm{Re}=3400$ in Figure 3. First column of Figure 3, instantenous velocity fields have very wavy structure coming from shedding vortices both side of the SCs. Due to lower pressure in the wake region, shedding vortices move toward the wake region. For $G / D=1.0$, the vortices interact vigorously. For $G / D=1.25$ and 1.50 , a jet like flow occurs between the square cylinders. This jet like flow travel toward one of the square, spontaneously even though the square cylinder has a fixed separation point at the front corner. Wake region of the oriented flow side becomes smaller than the other square cylinder. For $G / D=2.0$ and 3.0 , the obtained flow structures are very similar to single cylinder case. Time-averaged vorticity patterns $\langle\omega\rangle$ and Reynolds stress correlations $<\mathrm{u}^{\prime} \mathrm{v}^{\prime} / \mathrm{U}_{\infty}^{2}>$ for two side-by-side SCs for the gap spacing ratios in the range of $1.0<$ $G / D<3.0$ for $R e=3400$ are shown in the second and last column of Figure 3. Here, $G$ indicates the distance between SCs centers. The Time-averaged vorticity patterns $\langle\omega\rangle$ presented in the first row of Figure 3 indicate that a single wake region occurs downstream of side-by-side SCs in contact with one another. When $G / D=1.0$, the effective width of the two-body arrangement is a summation of each SC width. The vortex shedding frequencies of two SCs for $G / D=1.0$ are found as $0.587 \mathrm{~Hz}$ respectively which is about half of the frequencies of single SC $(1.114 \mathrm{~Hz})$ for $\mathrm{Re}=3400$. The flow characteristics of fluid-structure interactions become a strong function of side-by-side arrangements of the SCs until a certain gap ratio G/D. If the bodies are sufficiently close, two vortex streets interact vigorously, and flow features significantly differ from those of single vortex street downstream of an isolated bluff body. In the case of two side-by-side SCs, it is experimentally found that the wake flow structure is asymmetrical at small gap ratios such as $G / D=1.25$ and 1.5 , as a result of jet-like flow occurred between the SCs. The time- 
averaged inner vortices are too weak to affect the whole wake pattern for these gap ratios. That is to say, the two cylinder act like single bluff body, accompanied by a street of vortices shed at the free-stream sides. Despite the geometrical symmetry, the jet-like flow for $\mathrm{G} / \mathrm{D}=1.25$ and 1.5 cases is deflected towards the upper or lower side of the SC which has a narrow wake region and higher vortex shedding frequency and vice versa. Such observations are consistent with previous experimental results of $[1,8,12,14]$ for CCs. The length of the vortex pair downstream of upper SC persists around 2.7D in the downstream direction while the lower SC wake covers the whole image for $G / D=1.5$ presented in third row of Figure 3. Shedding vortices move in the flow direction without interfering with each other in the range of $2.0 \leq G / D \leq 3.0$. However, shedding vortices for the SCs interfere with each other until $G / D=2$. The vortex shedding frequency of two SCs is in close agreement with a single SC case when the gap ratio G/D is larger than 2.5. The Strouhal numbers for $\mathrm{G} / \mathrm{D}=3.0$ for two side-by-side SCs case for $\operatorname{Re}=3,400$ is calculated as 0.14 , which is agreed very well [18]. The vortex shedding frequency of single SC case is significantly higher than the other SC cases. Bearman and Wadcock [8] addressed that the jet-like flow was deflected to the high frequency side of the wake, and the cylinder on this side has a greater drag force than the other cylinder for the CCs cases.

Patterns of Reynolds stress correlations $\left\langle u^{\prime} v^{\prime} / U_{\infty}^{2}>\right.$ for different gap ratios are given in the last column of Figure 3 . They consist of both small-scale clusters located in close vicinity of the SC and large-scale clusters located just downstream of the small-scale clusters for $\mathrm{G} / \mathrm{D}=1.0$. A well-defined Reynolds stress patterns due to the velocity fluctuations along the shear layers are developed. More specifically, a weaker Reynolds stress region occurs close to the base of the SCs because of lower rate of entrainment occurred between core and wake flow regions as seen in the top row of Figure 3. A pair of large-scale Reynolds stress clusters exists immediately downstream of the small-scale Reynolds stress clusters for $G / D=1.0$. The magnitude of Reynolds stress correlations $\left\langle u^{\prime} v^{\prime} / U_{\infty}^{2}\right\rangle$ increase along both shear layers until the merging point of shear layers emanating from both sides of the cylinder. After that, the strength of the Reynolds stress correlations $<\mathrm{u}^{\prime} \mathrm{v}^{\prime} / \mathrm{U}_{\infty}^{2}>$ decays down in the direction of main flow. Patterns of same signed Reynolds stress correlations change their downstream locations from one side of the centerline of the image to the other side for $G / D=1.0$. Reynolds stress correlations are zero about centerline axis as a result of symmetrical flow structure and attains its highest magnitude in both sides of the centerline for $G / D=1.0$. Reynolds stress correlations $\left\langle u^{\prime} v^{\prime} / U_{\infty}^{2}\right\rangle$ in the downstream region of cylinders are very complex, unorganized and weaker than other cases for $G / D=1.25$ and 1.5. Even though, they are still detectable along the jet-like flow region for $G / D=1.25$ and $G / D=1.5$. The strength of the jet-like flow decreases with increasing gap ratio. Values of Reynolds stress correlations $\left\langle\mathrm{u}^{\prime} \mathrm{v}^{\prime} / \mathrm{U}_{\infty}^{2}\right\rangle$ become approximately zero along the centerline which passes through the SCs gap for the cases of $G / D>2.0$, which means that the net momentum transport across the centerline is negligible. These findings are similar to the results of Kolar et al. [18]. It is observed that flow patterns have considerable symmetry about the centerline of images for $G / D=3.0$. Since each of $S C$ acts like a single cylinder, shear layers around each cylinders do not interfere each other. The peak value of $\left\langle\mathrm{u}^{\prime} \mathrm{v}^{\prime} / \mathrm{U}_{\infty}^{2}\right\rangle$ is 0.158 for two $S C s$ at $G / D=3.0$ which is similar to the experimental results of the single square cylinder case having value of 0.15 conducted [18]. The patterns of Reynolds stress correlations $\left\langle\mathrm{u}^{\prime} \mathrm{v}^{\prime} / \mathrm{U}_{\infty}^{2}\right\rangle$ for two SCs and, in particular, for the range of $1.0<\mathrm{G} / \mathrm{D}<2.0$, are fundamentally different from the pattern for single SC case. The peak values of Reynolds stress correlations $\left\langle u^{\prime} v^{\prime} / U_{\infty}^{2}>\right.$ are significantly lower for $G / D=1.25$, which is found as 0.06 
for two side-by-side SCs. Wake size is larger than that of the single square cylinder and this wake size is decreased when the value of G/D is increased.
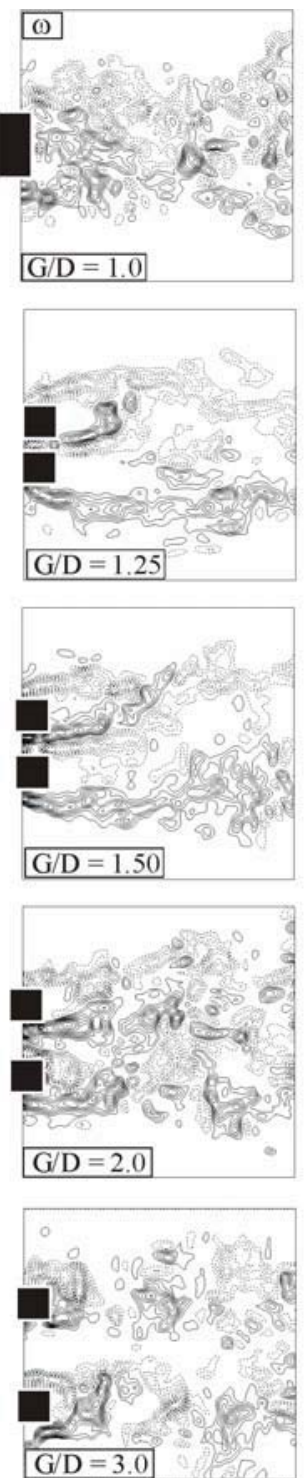
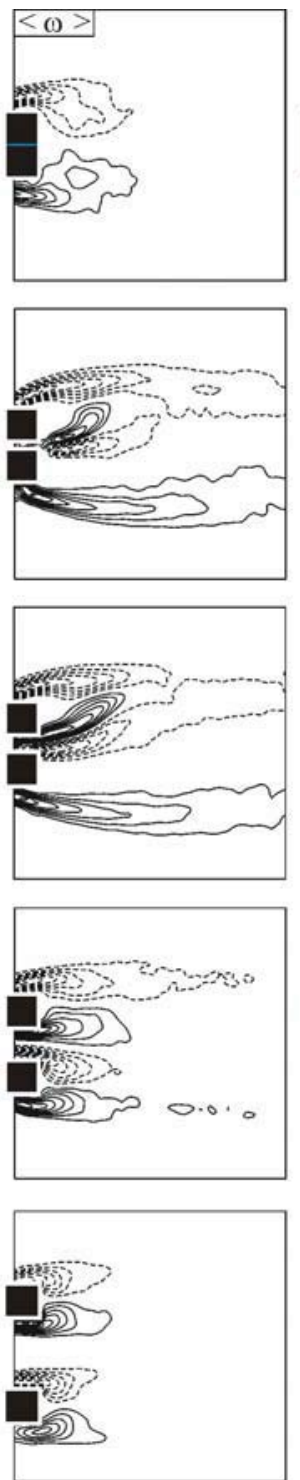
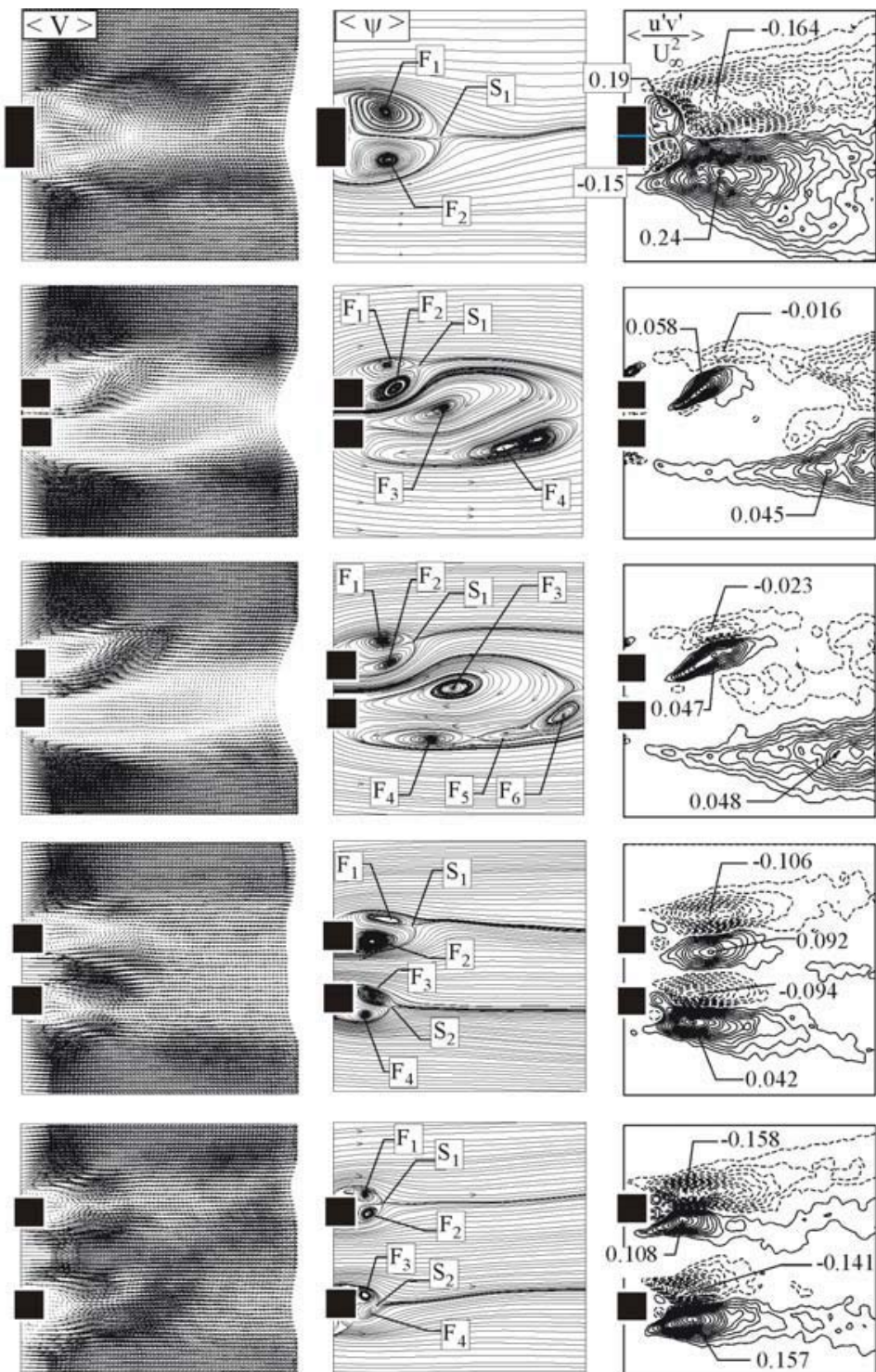
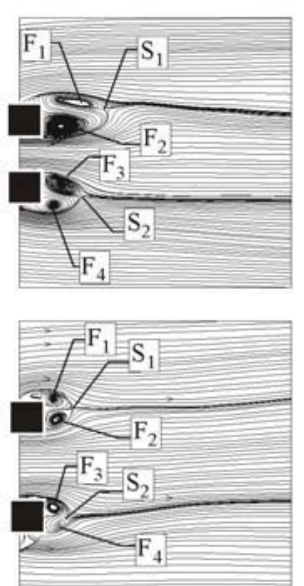
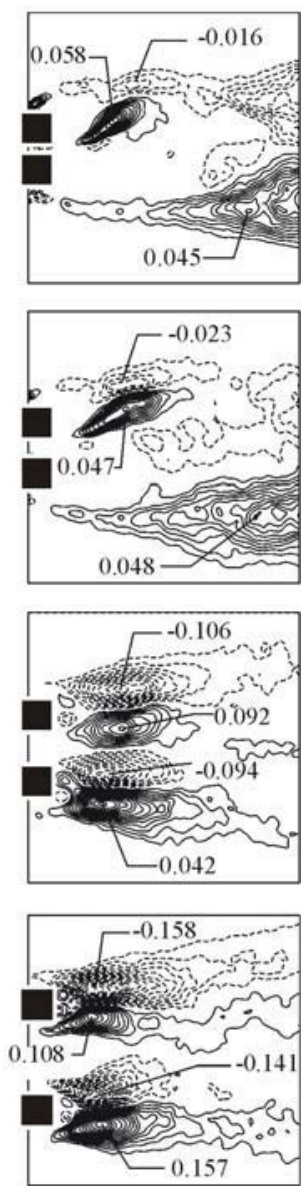

Figure 3: Comparison of instantaneous vorticity $\omega$, time-averaged vorticity contours $\langle\omega\rangle$, time-averaged velocity field $\langle\mathrm{V}\rangle$, streamline patterns $\langle\mathrm{y}\rangle$ and Reynolds stress correlations $\left\langle\mathrm{u}^{\prime} \mathrm{v}^{\prime} / \mathrm{U}_{\infty}^{2}\right\rangle$ as a function of gap spacing ratio $\mathrm{G} / \mathrm{D}=1.0$ to 3.0 is presented for two side-by-side $\mathrm{SCs}$ at $\mathrm{Re}=3,400$. Minimum and incremental contour values of vorticity are $\left.<\omega_{\min }\right\rangle= \pm 4 \mathrm{~s}^{-1}$ and $\langle\Delta \omega\rangle=4 \mathrm{~s}^{-1}$, respectively. Minimum and incremental contour values of Reynolds stress correlations $\left\langle\mathrm{u}^{\prime} \mathrm{v}^{\prime} / \mathrm{U}_{\infty}^{2}\right\rangle$ are $\left\langle\mathrm{u}^{\prime} \mathrm{v}^{\prime} / \mathrm{U}_{\infty}^{2}\right\rangle_{\min }=\left\langle\Delta \mathrm{u}^{\prime} \mathrm{v}^{\prime} / \mathrm{U}_{\infty}^{2}\right\rangle= \pm 0.01$ for $\mathrm{G} / \mathrm{D}=1.0,2.0$ and 3.0 and $\left\langle\mathrm{u}^{\prime} \mathrm{v}^{\prime} / \mathrm{U}_{\infty}^{2}\right\rangle \min =\left\langle\Delta \mathrm{u}^{\prime} \mathrm{v}^{\prime} / \mathrm{U}_{\infty}^{2}\right\rangle= \pm 0.005$ for $\mathrm{G} / \mathrm{D}=1.25$ and 1.50 , respectively. 
In the third and fourth columns of Figure 3 show the time-averaged velocity fields and streamline patters. Time-averaged velocity field clearly displays symmetrical flow structure for $G / D=1.0, G / D=2.0$ and $G / D=3.0$ as well as biased flow fields for $G / D=1.25$ and $G / D=1.50$. For $G / D=1$, the obtained flow structure are symmetrical and very similar to one given in Figure 2. The symmetrical flow structure for $G / D>1.00$ deteriorates with the effect of the jet-like flow occurred between the two square cylinders. For example, in the cases of $\mathrm{G} / \mathrm{D}=1.25$ and $\mathrm{G} / \mathrm{D}=1.50$, smaller foci $F_{1}$ and $F_{2}$ are developed in the wake region of upper square cylinder with a small bubble. Two bigger focus and a saddle point $S_{2}$ for $G / D=1.25$ cases occur further downstream region. For $G / D=1.50$, for different foci are observed in the wake of the bottom square cylinder. For the case of $G / D=2.0$ and 3.0, the jet-like flow becomes ineffective on the flow structures and hence the time-averaged patterns of streamline patterns $\langle\psi\rangle$ of two spheres are very similar to each other.

\subsection{Bistable flow structures for three side-by-side SCs}

Representative time-averaged vorticity contours $\langle\omega\rangle$ and streamline patterns $\langle\Psi\rangle$ for $\mathrm{Re}=1050$ and $\mathrm{Re}=2450$ for $\mathrm{G} / \mathrm{D}=1.25$ are shown in Figure 4 . The biased flow regimes have deflection angles toward lower or upper SC denoted as Case $A$ and Case B, respectively. Different deflection angles are observed for two and three side-by-side SCs as seen in Figure 4. For small gap spacing ratios, the flow does not remain deflected towards the same SC side continuously. That is to say sometimes, flow deflection occurs towards the upper or lower sides of SC for a long time period. This narrow wake which goes spontaneously toward the upper or lower SC in the downstream region of the centeral SC is called bistable flow structure confirmed by the previous experimental studies of $[8,9,13,14]$ for circular cylinders case. The results of these researches indicated that the bistable characteristic was not caused by misalignment of the circular cylinders or other extraneous influences, but was an intrinsic property of the flow. Recently, Sumner et al. (1999) for the circular cylinders have reported that a deeper underlying reason of the bistable is presently unknown [11]. The jet-like flow interacts with the vortex structure shed from three SCs and may induce bistable behavior of the near-wake flow. It is quantitatively observed that this bistable nature of the wake flow is not periodic. It is worth to note that the bistable flow structure can also persist by changing the free-stream velocity without changing the alignment of the SCs, even if the sharp-edged SC has constant separation points on the leading edge. The biased flow patterns for two side-by-side SCs are obtained for the gap values of $G / D \leq 1.25$. As seen in both cases $A$ and $B$, the bistable flow pattern is nominally independent of the Reynolds number. On one hand, the flow structure shown for $\operatorname{Re}=1050$ and $\operatorname{Re}=2450$ in Case $A$ indicates a wider wake flow region downstream of the upper SC, which corresponds to the smaller vortex shedding frequency. On the second hand, Case $B$ indicates a narrower wake flow region downstream of the upper SC corresponding to a higher vortex shedding frequency. The narrow vortex street on the biased side soon becomes indistinct and in engulfed by the large-scale vortex wake. Positive and negative vortices along the shear layers on the upper and lower sides of SCs extend for a longer distance comparing to the bias vortices. Further downstream of the SCs, both wake regions can merge to form a wider but weaker single wake flow region beginning approximately at 3.2D downstream of the SC. The time-averaged streamline patterns $\langle\psi\rangle$ clearly demonstrate the wake size and the flow direction. Patterns of the time-averaged streamline $\langle\psi\rangle$ exhibit well-defined critical points. For example, foci (centers of vortices) designated as $F$ and saddle points (apparent intersection of streamlines) as $S$. The focus $F_{1}$ located at the center of the wake region is stable which spirals inward direction. 



Figure 4: Bistable flow structures of representative time-averaged vorticity $\langle\omega\rangle$ and streamline patterns $\langle\Psi\rangle$ for $G / D=1.25$ are given for two different Re. Minimum and incremental values of positive and negative vortices are $\langle\omega \min \rangle= \pm 1.5 \mathrm{~s}^{-1}$ and $\langle\Delta \omega\rangle=$ $1.5 \mathrm{~s}^{-1}$ for $\operatorname{Re}=1050$ and $\left\langle\omega \min >= \pm 3 \mathrm{~s}^{-1}\right.$ and $\langle\Delta \omega\rangle=3 \mathrm{~s}^{-1}$ for $\operatorname{Re}=2450$, respectively. 
However, this focus $F_{1}$ is unstable and the vortex experiences contraction along its span direction which spirals in outward direction. The streamline patterns $\langle\psi\rangle$ associated with the formation of gap flow vortices, consisting of stable and unstable foci $F$ and saddle points $\mathrm{S}$, has displayed on the image in Figure 4. For $\mathrm{Re}=1050$ and $\mathrm{Re}=2450$, the domain of streamline patterns $\langle\psi\rangle$ of foci covers whole wake flow region. The time-averaged streamline patterns $\langle\psi\rangle$ indicate that five foci $\left(F_{1}\right.$ through $\left.F_{5}\right)$ and saddle points $S$ are evident at $\operatorname{Re}=1050$ for Case $A$ and $B$. Perry and Steiner (1987) and Delery (1992) pointed out that a spiral-type streamline patterns at the center of the vortex was an indicator of spanwise three-dimensionality of vortex structure. As shown in Figure 4, the spiral-type streamline patterns $\langle\psi\rangle$ are also observed. These streamline patterns $\langle\psi\rangle$ also indicate the size of the wake flow region clearly. Because of asymmetric flow, streamline patterns $\langle\psi\rangle$ originating from the wide wake region diverges broadly in the main flow direction further downstream of all SCs.

\section{CONCLUSIONS}

Interference of wake structure between two or three cylinders involves most of the generic flow features associated with multiple structures, thus providing an excellent model for gaining physical insight into the wake of multiple cylindrical structures. This study aims to provide an experimental systematic study of the flow behind single, two and side-by-side square cylinders. The square cylinder is a representative model for bluff bodies with sharp corners, characterized by a fixed flow separation point, which are distinct from those of continuous curvature with oscillating separation points, typically represented by the circular cylinder [22]. Experiments were performed at a Reynolds number Re of 1050, 2450 and 3400 and a cylinder centre-to-centre gap ratio G/D of 1.0-3.00. Following results are obtained;

- Relevant images for side-by-side arrangements obviously illustrate that the timeaveraged flow patterns are strong function of the gap spacing rather than Reynolds number. However, depending on Reynolds number, the wake region of one of the SCs can be sometimes larger than that of the other one even though having fixed separation point.

- For two side-by-side SCs, time-averaged vortices and Reynolds stress correlations $<\mathrm{u}^{\prime} \mathrm{v}^{\prime} / \mathrm{U}_{\infty}^{2}>$ exhibit symmetrical flow patterns with respect to centerline of the SCs in contact with one another for $G / D=1.0$ for $1050 \leq R e \leq 3400$. For two side-by-side SCs case, the flow structures behind the SCs are asymmetric (i.e.biased) at small gap ratios such as $G / D=1.25$ and $G / D=1.5$ as a result of jet-like flow between the SCs. The jet-like flow tends to deflect towards the narrow wake region that has a higher vortex shedding frequency. The interactions between the narrow and the wide wake regions are likely to lead to the early annihilating of the narrow wake and, meanwhile, prolong the formation process of a wider vortex row. The deflection angle of the gap flow reduces as the gap ratio enlarges.

- For the gap ratio $G / D=2.0$, influence of the jet-like flow on wake structures still exists for all side-by side SCs cases. For the gap ratios $G / D \geq 3.0$, the jet-like flow effect on the flow characteristics of two SCs cases is such a negligible level that the flow structure in the downstream of each SC is very much similar to single SC.

- The flow patterns have considerable symmetry about the centerline of the square cylinders of the flow patterns, in all time-averaged images for $R e=3400$ for both single and two side-by-side square cylinders gap ratio of $G / D=3.0$.

- Contours of the time-averaged streamwise velocity demonstrate that the stagnation point around the symmetry plane moves further upstream for higher $R e$. 
- Time-averaged cross-stream velocity contours and Reynolds stress correlations, which are oriented in the direction of each respective shear layer separation from the cylinder, are important features of the wake structures from the point of momentum transfer.

- Strouhal numbers for the single square cylinder for $1050 \leq \operatorname{Re} \leq 3400$ are found in the range of $0.12-0.13$. The present results have supported the previous works by providing detailed quantitative experimental information with PIV in the wake region of the SC.

\section{ACKNOWLEDGEMENT}

The authors would like to acknowledge the funding of Coordinatorship of Selcuk University's Scientific Research Contract No: 11401058 and Scientific Research Projects Office of Cukurova University under contract No: AAP20025.

\section{REFERENCES}

[1] Williamson, C.H.K., 1985. Evolution of a single wake behind a pair of bluff bodies. J. Fluid Mech., 159, 1-18.

[2] Okajima, A., 1982. Strouhal numbers of rectangular cylinders., J. Fluid Mech. 123, 379-398.

[3] Obasaju, E.D., 1983. An investigation of the effects of incidence on the flow around a square section cylinder. Aeronautical Quarterly 34, 243-259.

[4] Lyn, D.A., Einav, S., Rodi, W., Park, J.H., 1995. A laser-Doppler velocimetry study of ensemble-averaged characteristics of turbulent near wake of a square cylinder. J. Fluid Mech. 304, 285-319.

[5] Saha, A.K., Muralidhar, K., Biswas, G., 2000. Experimental study of flow past a square cylinder at high Reynolds numbers. Exp. in Fluids, 29, 553-563.

[6] Ozgoren, M., 2006. Flow structure in the downstream of square and circular cylinders, Flow Meas. and Inst., 17:225-235.

[7] Zdravkovich, M., 1977. Review of flow interference between two circular cylinders in various arrangements. ASME J. Fluids Eng. 99, 618-633.

[8] Bearman, P.W., Wadcock, A.J., 1973. The interaction between a pair of circular cylinders normal to a stream. J. Fluid Mech. 61, 499-511.

[9] Kim, H.J., Durbin, P.A., 1988. Investigation of the flow between a pair of circular cylinders in the flopping regime. J. Fluid Mech. 196, 431-448.

[10] Guillaume, D.W., LaRue, J.C., 1999. Investigation of the flopping regime with two-, three- and four-cylinder arrays. Exp. in Fluids 27, 145-156.

[11] Sumner, D., Wang, S.S.T., Price, S.J., Paidoussis, M.P., 1999. Fluid behavior of side-by- side circular cylinders in steady cross-flow. J. Fluids and Struct. 13, 309338.,

[12] Zhou, Y., Zhang, H.J., Yiu, M.W., 2002. The turbulent wake of two side-by-side circular cylinders. J. Fluid Mech. 458, 303-332.

[13] Zhou, Y., 2003. Vortical structures behind three side-by-side cylinders. Exp. in Fluids 34, 68-76. 
[14] Akilli, H., Akar, A., Karakus, C., 2004. Flow characteristics of circular cylinders arranged side-by-side in shallow water. Flow Meas. and Inst. 15, 187-197.

[15] Sayers, A.T., 1991. Steady-state pressure and force coefficients for groups of three equispaced square cylinders situated in a cross flow. J. Wind Eng. and Ind. Aerodyn. 37, 197-208.

[16] Miau, J.J., Wang, G.Y., Chou, J.H., 1994. Intermittent switching of gap flow downstream of two flat plates arranged side-by-side. J. Fluid Struct. 6, 563-582.

[17] Higuchi, H., Lewalle, J., Crane, P., 1994. On the structure of a two-dimensional wake behind a pair of flat plates. Physics of Fluids 6, 297-305.

[18] Kolar, V., Lyn, D.A., Rodi, W., 1997. Ensemble-averaged measurements in the turbulent near wake of two side-by-side square cylinders. J Fluid Mech. 346, 201237.

[19] Alam, Md.M., Moriya, M., Sakamoto, H., 2003. Aerodynamic characteristics of two side-by-side circular cylinders and application of wavelet analysis on the switching phenomenon. J.of Fluids and Struct. 18, 325-346.

[20] Bearman, P.W., Wadcock, A.J., 1973. The interaction between a pair of circular cylinders normal to a stream. J. Fluid Mech. 61, 499-511.

[21] Alam, Md.M., Moriya, M., Sakamoto, H., 2003. Aerodynamic characteristics of two side-by-side circular cylinders and application of wavelet analysis on the switching phenomenon. J.of Fluids and Struct. 18, 325-346.

[22] Alam, Md.M., Zhou Y. and Wang X.W., 2010. The wake of two side-by-side square cylinders, J. Fluid Mech., oi:10.1017/S0022112010005288, 1-40.

[23] Westerweel, J., 1993. Digital particle image velocimetry, theory and application. Delft University Press.

[24] Perry, A.E., Steiner, T.R., 1987. Large-scale vortex structures in turbulent wakes behind bluff bodies: Part 1 Vortex formation processes. J. Fluid Mech. 174, 233270.

[25] Delery, J.M., 1992. Physics of vortical flows. J. Aircraft 29, 856-876. 